\title{
Extraction, Partial Purification and Detection of Antimicrobial Metabolites Produced by the Rhizobacterial Strain UPMP3 of Pseudomonas aeruginosa and UPMB3 of Burkholderia cepacia and their Antagonistic Activity against Ganoderma boninense In vitro
}

\author{
Waheeda Parvin ${ }^{1,2 *}$, Md Mahbubur Rahman ${ }^{1,3}$ and Mui Yun Wong,4 \\ ${ }^{1}$ Bangladesh Forest Research Institute, Chattogram, Bangladesh \\ ${ }^{2}$ Department of Plant Protection, Faculty of Agriculture, Universiti Putra Malaysia, \\ Serdang, Malaysia \\ ${ }^{3}$ Department of Biochemistry, Faculty of Biotechnology and Biomolecular Sciences, \\ Universiti Putra Malaysia, Serdang, Malaysia \\ ${ }^{4}$ Institute of Plantation Studies, Universiti Putra Malaysia, Serdang, Malaysia \\ *Corresponding Author: Waheeda Parvin, Silviculture Genetics Division, \\ Bangladesh Forest Research Institute, Chattogram, Bangladesh.
}

Received: July 31, 2021

Published: August 13, 2021

(C) All rights are reserved by Waheeda

Parvin., et al.

\begin{abstract}
Antimicrobial metabolites are produced as secondary metabolites by microorganism as well as the plant growth promoting rhizobacteria. These compounds are widely distributed in nature, where they play an important role in regulating the microbial population of soil, water, sewage and compost. In the present investigation, some antimicrobial metabolites such as antibiotics, siderophores, and HCN were detected in vitro by TLC, CAS agar universal test plates and filter paper with alkaline picrate solution method respectively from the two rhizobacterial strains Pseudomonas aeruginosa UPMP3 and Burkholderia cepacia UPMB3. In vitro bioassay was carried out through antagonistic activity test against $G$. boninense based on the percentage inhibition of radial growth (PIRG). The strains showed antifungal activity against Ganoderma boninense that is responsible for the disease of basal stem rot in oil palm. The crude extracts obtained from ethyl acetate solvent extraction and analyzed by thin layer chromatography (TLC). Six different antibiotics were detected with different retention factors $\left(\mathrm{R}_{f}\right)$ on TLC plates. The $\mathrm{R}_{f}$ values were calculated as $0.88,0.77,0.63,0.53$, $0.47,0.28$ and 0.23 for 2-4 DAPG, pyoluteorin, phenazine, pyocyanin, phenazine-1-carboxamide (PCN) and pyrrolnitrin successively developed with different solvent systems. Among the different solvent systems ethyl acetate: chloroform was most effective in separating the active bands from the extracts. Siderophores were detected through colour change into blue to orange and HCN was in dark brown to red colour. The antagonistic activity of Pseudomonas aeruginosa UPMP3 and Burkholderia cepacia UPMB3 was evaluated. In case of bacterial antibiotics and volatile and non-volatile effects, the strain P. aeruginosa UPMP3 showed the highest $94.21 \%$ and $51.16 \%$ inhibitory on the mycelial growth of $G$. boninense than B. cepacia UPMB3 (21.27\% and 8.89\%) compared to control treatment after 7 days of incubation respectively. The findings of this study indicate that these two rhizo-bacterial strains are capable to control Ganoderma boninense through producing antimicrobial metabolites.
\end{abstract}

Keywords: Purification; Detection; Antifungal Metabolites; Pseudomonas aeruginosa UPMP3; Burkholderia cepacia UPMB3; Antagonistic Activity; Ganoderma boninense, In Vitro

\section{Abbreviations}

PGPR: Plant Growth-Promoting Rhizobacteria; P.a.: Pseudomonas aeruginosa; B.c.: Burkholderia cepacia; G.b.: Ganoderma boninense;
PDA: Potato Dextrose Agar; KB: King's B Agar; NB: Nutrient Agar; MEA: Malt Extract Agar; CAS: Chrome Azurol Sulphonate, HDTMA: Hexa Decyltrimethylammonium Bromide; TLC: Thin Layer Chro- 
matography; HCN: Hydrogen Cyanide; 2,4-DAPG: 2, 4-diacetylphloroglucinol; PLT: Pyoluteorin, PRN: Pyrrolnitrin; PCN: Phenazine-1 - Carboxamide, PCA: Phenazine -1 - Carboxylic Acid; PIRG: Percentage Inhibition of Radial Growth; CRD: Completely Randomized Design; LSD: Least Significant Difference; MPOB: Malaysian Palm Oil Board

\section{Introduction}

The plant growth-promoting rhizobacteria (PGPR) improve plant growth either directly via production of plant growth regulators such as auxins and cytokinin's and by increasing the plant uptake of some micro and macro elements in the rhizosphere or indirectly, through biological control of pathogens or induction of host defense mechanisms. Production of antifungal secondary metabolites, such as antibiotics, hydrogen cyanide (HCN), siderophore and lytic enzymes is a prominent feature of many biocontrol fluorescent pseudomonads. There are several bacterial antibiotics which to play a central role in disease management. A variety of antibiotics have been identified, including: amphisin, 2,4-diacetylphloroglucinol (2,4-DAPG), oomycin A, phenazine, pyoluteorin (PLT), pyrrolnitrin (PRN), tensin, tropolone, and cyclic lipopeptides produced by pseudomonads and Oligomycin A, kanosamine, zwittermicin A, and xanthobaccin were found to be produced by Bacillus, Streptomyces, and Stenotrophomonas spp respectively [1,2]. The first antibiotics described as being implicated in biocontrol were phenazine derivatives produced by fluorescent pseudomonas [3]. The axenic culture of Peudomonas aeruginosa strain UPMP3 has shown to produce core phenazine (PHZ), phenazine-l-carboxylic acid (PCA) and pyocyanin (PYO) antibiotics at variable concentrations and those antibiotics were able to inhibit the growth of Ganoderma boninense in vitro [4]. Production of antibiotics is closely related to the overall metabolic system of the organism, which in turn is dictated by nutrient availability and other environmental stimuli, such as type of carbon source and supply, major and minor minerals, $\mathrm{pH}$, temperature, and other parameters [5]. The varied arsenal of bio antagonistic strains may enable the pathogen to perform their ultimate objective of disease suppression under different environmental conditions. For example, in $P$. fluorescens $\mathrm{CHA0}$ biosynthesis of DAPG is stimulated and pyoluteorin is repressed in the presence of glucose as a carbon source. As glucose is depleted, however, pyoluteorin becomes the more abundantly antimicrobial compound produced by this strain. This ensures a degree of flexibility for the antagonist when confronted with a different or a changeable environment. Biotic environment can also effect pro- duction of antibiotics [6]. Secondary metabolites e.g. salicylates and pyoluteorin can influence DAPG produced by $P$. fluorescens CHA0. Furthermore, plant development and growth also influence antibiotic synthesis [7]. Since biological activity of DAPG producers strain is not affected by the exudates of young plant roots but is induced by the exudates of older plants, which results in selective pressure against other microflora.

Plant growth promoting bacteria produce low molecular weight compounds, under iron limiting condition, called siderophores to competitively acquire ferric ion. Although various bacterial siderophores differ in their abilities to sequester iron, in general, they deprive pathogenic fungi of this essential element since the fungal siderophores have lower affinity. Lodewyckx., et al. [8] stated that some plant growth promoting bacteria draw iron from heterologous siderophores excreted by other soil microflora. The production of siderophores is an indirect mechanism associated with the increase in plant growth by PGPR. The production of bacterial siderophores stimulates plant growth by increasing iron availability in the rhizosphere and inhibiting pathogen growth in the rhizosphere [9]. Siderophores are produced by Pseudomonas bacteria to compete for iron and consequently impairing growth of soil-borne phytopathogens, and thus are considered as a control mechanism for many pathogens. In addition, in vitro assays showed that the inhibition of pathogens based on competition for iron tends to decrease with increasing iron content of the medium [10].

Volatile and non-volatile compounds such as HCN, ammonia, aldehydes, aliphatic alkanes, alcohols, organic acids, ketones, fatty acid, gasoline etc. are produced by many rhizosphere strains and have been implicated as important metabolites in biocontrol. The use of such PGPR producing phytohormones and antimicrobial metabolites is a new concept to solve the replant problem to some extent. More specifically, the soil-borne Pseudomonas sp. has received particular attention because of their capacity to produce a wide range of phytohormones, and antimicrobial metabolites. PGPR produce HCN which depend on soil and plant characteristics. It is a volatile secondary metabolite that suppresses the development of microorganisms and also affects negatively the growth and development of plants, if produce in excess amount [11]. HCN first inhibits the electron transport and the energy supply to the cell is disrupted leading to the death of the organisms. To date many different bacterial genera have shown to produce $\mathrm{HCN}$, including species of Alcaligenes, Aeromonas, Bacillus, Pseudomonas and Rhizobium [12]. Volatile and nonvolatile compounds may also contribute

Citation: Waheeda Parvin., et al. "Extraction, Partial Purification and Detection of Antimicrobial Metabolites Produced by the Rhizobacterial Strain UPMP3 of Pseudomonas aeruginosa and UPMB3 of Burkholderia cepacia and their Antagonistic Activity against Ganoderma boninense In vitro". Acta Scientific Agriculture 5.9 (2021): 17-29. 
to inhibition of growth of fungal pathogens. Indeed, several studies have shown the importance of volatiles and nonvolatiles in the biocontrol of different plant diseases. Production of volatiles and nonvolatiles in liquid cultures proved inhibitory to spore germination and mycelial growth [13].

\section{Aim of the Study}

The aim of this study was to detect important antimicrobial metabolites (antibiotics, siderophores, and HCN) produced by Pseudomonas aeruginosa UPMP3 and Burkholderia cepacia UPMB3 isolated from oil palm rhizosphere and to evaluate the suppression of G. boninense in vitro.

\section{Materials and Methods}

\section{Sources of the bacterial strains}

Two plant growth promoting rhizobacteria Pseudomonas aeruginosa UPMP3 and Burkholderia cepacia UPMB3 were collected from Plant Protection Department, Universiti Putra Malaysia. These bacterial strains were isolated from oil palm rhizosphere. Pseudomonas aeruginosa strain UPMP3 is a $\gamma$ Proteobacterium and Burkholderia cepacia strain UPMB3 is a $\beta$ Proteobacterium [14]. Both strains have been characterized, sequenced and deposited with NCBI Gen Bank (P. aeruginosa strain UPMP3 -Accession no. GQ183951 and B. cepacia strain UPMB3 - GQ183952) by Azadeh and Sariah [15]. In the current study, these bacterial strains were prepared from stock cultures stored at $4^{\circ} \mathrm{C}$ and subsequently sub-cultured on nutrient agar when required. The bacterial strains were also identified based on Biolog ${ }^{\circledR}$ identification system [14].

\section{Detection of antibiotics}

Preparation of bacterial strains

P. aeruginosa UPMP3 and B. cepacia UPMB3 $\left(10^{8} \mathrm{cfu} / \mathrm{mL}\right)$ were cultured in KB (Kings B agar) medium and incubated at $28 \pm 2{ }^{\circ} \mathrm{C}$ for 24 hours. Then the bacterial strains were cultured in King's B broth. The initial $\mathrm{pH}$ of broth medium was adjusted to 7.0. The experiment was carried out in $500 \mathrm{~mL}$ Erlenmeyer flasks containing $200 \mathrm{~mL}$ of the medium and the medium was inoculated with 2 loops of 24 hours pre-cultured bacterial strains separately. Inoculated flasks were incubated at $28 \pm 2{ }^{\circ} \mathrm{C}$ on an incubator shaker at 170 rpm for 5 days.

Extraction of antibiotics from $P$. aeruginosa UPMP3 and $B$. cepacia UPMB3

The bacterial culture was centrifuged at $10,000 \mathrm{rpm}$ for 20 minutes after 5 days of incubation. The pellet was discarded and the supernatant was acidified to $\mathrm{pH} 2.5$ with $1 \mathrm{~N} \mathrm{HCl}$. Then the supernatant was centrifuged again at $8,000 \mathrm{rpm}$ for another $10 \mathrm{~min}$ and the precipitate was discarded. The final $\mathrm{pH}$ of the supernatant was adjusted to 7.0 with phosphate buffer $(1 \mathrm{M}, \mathrm{pH}$ 8). This solution was designated as crude supernatant [16]. For the extraction of different antibiotics, different solvents were used. Equal volume of benzene, ethyl acetate and chloroform were used for the extraction of antibiotic phenazine, 2, 4 Diacetyl phloroglucinol and pyocyanin respectively. Double volume of ethyl acetate was used for Phenazine -1 - Carboxamide (PCN). 80\% acetone was used for pyoluteorin and pyrrolnitrin. Each fraction was evaporated separately and dried. The antibiotics were dissolved in 1- $3 \mathrm{~mL}$ of absolute methanol and purified with $0.22 \mu \mathrm{m}$ membrane filter. These partial purified stocks were used for the detection of antibiotics through TLC analysis.

Thin layer chromatography analysis

The purified antibiotic stocks were spotted on TLC plates and run with different solvent systems to detect the antibiotics. $10 \mu \mathrm{L}$ of each stock were used for spotting. Different solvent systems i.e. toluene: acetone (4:1), chloroform: acetone (9:1), isopropanol: ammonia: water (8:1:1), ethyl acetate: chloroform (9:1), hexane: ethyl acetate (3:2), acetonitrile: methanol: water $(1: 1: 1)$ were used to detect different antibiotics. TLC plates were visualized under UV light at $254 \mathrm{~nm}$ and $365 \mathrm{~nm}$ wavelength and bands were detected. $\mathrm{R}_{f}$ values of the detected bands were compared with $\mathrm{R}_{f}$ values of antibiotics extracted from reference strains.

\section{Detection of siderophores}

Bacterial strains and media preparation

P. aeruginosa UPMP3 and B. cepacia UPMB3 $\left(10^{8} \mathrm{cfu} / \mathrm{mL}\right)$ were cultured in nutrient agar or King's B agar medium and incubated at $28 \pm 2{ }^{\circ} \mathrm{C}$ for 24 hours. To detect and optimize siderophores production, both the bacterial strains were cultured in three different culture media i.e. Chrome Azurol S (CAS, Sigma Aldrich), Nutrient agar combined with CAS (NA + CAS) and King's B agar combined with CAS $(\mathrm{KB}+\mathrm{CAS})$ medium.

Preparation of modified CAS agar universal test plates

The universal CAS assay was modified to test the ability of the bacterial strains to produce siderophores. CAS-blue agar medium (1L) was prepared according to Schwyn and Neilands [17] using $60.5 \mathrm{mg}$ CAS dissolved in $50 \mathrm{~mL}$ water distilled, deionized, and mixed with $10 \mathrm{~mL}$ iron (III) solution $\left(1 \mathrm{mM} \mathrm{FeCl}_{3} \cdot 6 \mathrm{H}_{2} \mathrm{O}, 10 \mathrm{mM}\right.$

Citation: Waheeda Parvin., et al. "Extraction, Partial Purification and Detection of Antimicrobial Metabolites Produced by the Rhizobacterial Strain UPMP3 of Pseudomonas aeruginosa and UPMB3 of Burkholderia cepacia and their Antagonistic Activity against Ganoderma boninense In vitro". Acta Scientific Agriculture 5.9 (2021): 17-29. 
$\mathrm{HCl}$. Under stirring, this solution was slowly added to $72.9 \mathrm{mg}$ HDTMA (Hexa decyltrimethylammonium bromide) dissolved in 40 $\mathrm{mL}$ water. The resultant dark blue liquid was autoclaved at $121^{\circ} \mathrm{C}$ for $15 \mathrm{~min}$. Another mixture of $750 \mathrm{~mL}$ water, $15 \mathrm{~g}$ agar, $30.24 \mathrm{~g}$ Pipes, and $12 \mathrm{~g}$ of a solution of $50 \%(\mathrm{w} / \mathrm{w}) \mathrm{NaOH}$ was autoclaved to raise the $\mathrm{pH}$ to the $\mathrm{pKa}$ of Pipes (6.8). The dye solution was finally poured along the glass wall and agitated with enough care to avoid foaming. Petri dishes (10 $\mathrm{cm}$ in diameter) were prepared with 30 $\mathrm{mL}$ of appropriate medium to culture each strain. After solidification, the CAS medium was cut into halves $(15 \mathrm{~mL})$, one of which was replaced by NA medium to prepare NA + CAS agar plates. Similarly, to prepare $\mathrm{KB}+\mathrm{CAS}$ plate halves $(15 \mathrm{~mL})$ was replaced by KB medium.

\section{Bacteria culture and observations}

The bacterial strains were cultured on each different plates mentioned above to observe the response of siderophores production. One loop of pre-cultured bacterial strains $\left(10^{8} \mathrm{cfu} / \mathrm{mL}\right)$ was streaked on CAS agar plate only. In the case of combined media plates NA + CAS and KB + CAS, the pre-cultured inoculum was placed as far as possible from the borderline between the two media. The plates were incubated at $30^{\circ} \mathrm{C}$ temperature for 3 weeks in the dark. The growth rate of strains were daily monitored and expressed as the number of days required by the bacteria to cover the halves of petriplates containing the culture medium. The CAS reaction rate was determined by measuring the advance of the colorchange in the CAS-blue agar, starting from the borderline between the two media. The CAS-agar color changed blue to orange. The control plates of CAS-agar and combined CAS agar un-inoculated were incubated under the same conditions as described above and no color change in the CAS-blue agar was observed, even after long incubation periods (3-4 weeks).

\section{Detection of hydrogen cyanide}

Bacterial strains and growth conditions

P. aeruginosa UPMP3 and B. cepacia UPMB3 was used to detect HCN production. Bacterial strains were cultured in King's B agar medium and incubated at $28 \pm 2^{\circ} \mathrm{C}$ for 24 hours.

\section{Media preparation for $\mathrm{HCN}$ production}

Production of HCN from the both bacterial strains was detected using the method of Reddy., et al. [18] with few modifications. Pre cultured bacterial strains $\left(10^{8} \mathrm{cfu} / \mathrm{mL}\right)$ were grown in sterilized test tubes containing $10 \mathrm{~mL}$ of King's B broth medium supplement- ed with $4.4 \mathrm{gm} / \mathrm{L}$ glycine and incubated for 48 hours at $28 \pm 2^{\circ} \mathrm{C}$ at $120 \mathrm{rpm}$ on incubator shaker. Filter paper (Whatman No. 1) strips of uniform size $(10 \mathrm{~cm} \times 0.5 \mathrm{~cm})$ soaked in $1.3 \%$ alkaline picrate solution (2.5 g picric acid and $2.5 \mathrm{~g} \mathrm{Na}_{2} \mathrm{CO}_{3} / \mathrm{L}$ ) and placed inside the test tubes in hanging position and closed with cap. Then the test tubes were wrapped with parafilm and incubated at room temperature $\left(28 \pm 2^{\circ} \mathrm{C}\right)$ for 7 to 10 days. After incubation, the sodium picrate present in the filter paper was reduced yellow to reddish compound in proportion to the amount of HCN acid evolved. Un-inoculated broth medium was used as control.

In vitro bioassay of antimicrobial compounds against Ganoderma boninense

Antimicrobial metabolites HCN, siderophores, and different antibiotics (phenazines, pyocyanin, pyrrolnitrin, 2,4-diacetylphloroglucinol, pyoluteorin etc.) produced by the two PGPR P. aeruginosa UPMP3 and B. cepacia UPMB3 have been detected in this study. These bacterial strains were then screened for their antagonistic activity test against $\mathrm{G}$. boninense in vitro based on the percentage inhibition of radial growth (PIRG).

\section{Preparation of bacterial strains and $G$. boninense}

Both the bacterial strains were cultured and maintained on $\mathrm{Nu}-$ trient agar (NB) or King's B (KB) agar media. The culture plate of the pathogen $G$. boninense, strain PER71 was first obtained from Malaysian Palm Oil Board (MPOB). It was maintained and stored at the pathology lab, Department of Plant Protection, Faculty of Agriculture, Universiti Putra Malaysia. After that it was collected from pathology lab and used for bioassay of antifungal activity. The method was adapted from Zaiton., et al. [14]. The culture was sub-cultured on Malt Extract Agar (MEA, Merck) medium at $28^{\circ} \mathrm{C}$ for 8 to 9 days until the mycelium had fully covered the whole agar plate. The Ganoderma culture agar plates were checked daily to examine the growth of mycelium and if there were any bacterial or fungal contamination. Culture with crust after 6 to 7 days of incubation was considered as good inoculum and used for bioassay.

\section{Antagonistic activity test}

To evaluate the antagonistic activity of the bacterial strains, a $5 \mathrm{~mm}$ diameter agar disc was taken from the 7 days-old MEA culture of $G$. boninense and plugged centrally in KB agar plate and then one loop of pre cultured bacterial strains were streaked $3 \mathrm{~cm}$ away from $G$. boninense plug. All the antagonistic pairings were incubated at $28 \pm 2 \stackrel{\circ}{\circ}$. The PIRG was obtained after 7 days incubation.

Citation: Waheeda Parvin., et al. "Extraction, Partial Purification and Detection of Antimicrobial Metabolites Produced by the Rhizobacterial Strain UPMP3 of Pseudomonas aeruginosa and UPMB3 of Burkholderia cepacia and their Antagonistic Activity against Ganoderma boninense In vitro". Acta Scientific Agriculture 5.9 (2021): 17-29. 
The ability of the bacteria to inhibit the growth of $G$. boninense was assessed after 7 days incubation by measuring the radius of the $G$. boninense colony in the direction towards the antagonist colony (R2). The data were later transformed into percentage inhibition of radial growth (PIRG) in relation to the radial growth of $G$. boninense in the control plate (R1) using the formula:

$$
\operatorname{PIRG}(\%)=\frac{\mathrm{R} 1-\mathrm{R} 2}{\mathrm{R} 1} \times 100
$$

R1= Radius of the $G$. boninense colony in the control plate

$\mathrm{R} 2=$ Radius of the $G$. boninense colony in the dual culture plate.

According to Zaiton., et al. [14], the antagonistic activities of these two bacteria have been determined.

\section{In vitro bioassay of antibiotics against $G$. boninense}

In vitro bioassay of antibiotics was carried out according to Montealegro., et al. [19] with modification instead of PDA plates; King's B plates were used. The plates containing King's B agar medium were covered with a cellulose nitrate membrane and the bacterial suspension $\left(10^{8} \mathrm{cfu} / \mathrm{mL}\right)$ of King's B broth was inoculated in the centre. After 48 hours incubation at $28 \pm 2^{\circ} \mathrm{C}$, the membrane with the grown bacterial strains was removed. The plates were inoculated with a $5 \mathrm{~mm}$ disk of 5 days pure culture of $G$. boninense in the middle and re-incubated at room temperature for 7 days. The growth of the pathogen was measured. The un-inoculated King's B plates containing the cellulose nitrate membrane were used as control (replacing the bacterial suspension by sterile distilled water), and further incubated with pathogen $G$. boninense. Results were expressed as mean \% of inhibition of $G$. boninense in the presence and absence of antagonistic bacterial strains respectively. Percentage of inhibition was calculated using the formula according to Zaiton., et al [14].

In vitro bioassay of volatiles and non-volatiles against $G$. boninense

In vitro bioassay of volatile and non-volatile antimicrobial metabolites production was carried out by the antagonistic bacterial strains according to Montealegro., et al. [19]. Two half petriplates (sterile) were taken and were poured with sterile molten and cooled King's B agar medium. The medium was allowed to solidify. Then the 24 hours pre-cultured bacteria were inoculated on one of the half plate in centre and on the other half plate was inoculated with 5 days old pure cultured Ganoderma. Both half plates were placed face to face preventing any physical contact between the pathogen and the bacterial strains. The plates were sealed with parafilm to maintain the inside atmosphere and to prevent loss of volatiles and non-volatiles produced. Plates were then incubated at $28 \pm 2^{\circ} \mathrm{C}$ for 7 days. Growth of the pathogen Ganoderma was measured and compared to the control plates prepared alone with Ganoderma. Results are calculated as mean (\%) of inhibition of the growth of fungal pathogens in the presence and absence of bacterial strains. Percentage of inhibition was calculated using the formula according to Zaiton., et al [14].

\section{Statistical analysis}

All experiments were performed as Completely Randomized Design (CRD). Data were analysed using statistical analysis system (SAS v9.3) and means were statistically compared using LSD test. The significance level was set up at $\mathrm{p}<0.05$. Three replications were considered for each treatment and repeated twice.

\section{Results and Discussion}

\section{Detection of antibiotics}

Phenazine and other antibiotics in the crude extract produced by the two bacterial strains P. aeruginosa UPMP3 and B. cepacia UPMB3 were determined by TLC analysis. Six different antibiotics were detected with different retention factors $\left(\mathrm{R}_{f}\right)$ on TLC plates under UV light at $365 \mathrm{~nm}$. The $\mathrm{R}_{f}$ values were calculated as 0.88 , $0.77,0.63,0.53,0.47,0.28$ and 0.23 developed with different solvent systems. The chromatographic properties of these antibiotics were compared with those of known compounds from reference strains. Results are presented in figure 1 . The isolated compounds behaved similar to the references when compared by TLC. Among the different solvent systems ethyl acetate: chloroform was most effective in separating the active bands from the extracts. In this solvent system, extracted phenazine and pyocyanin from strains $P$. aeruginosa UPMP3 absorbed and appeared as blue bands with $\mathrm{R}_{f}$ value 0.63 which was identical in reference to $R_{f}$ value of phenazine extracted from Pseudomonas sp. [20]. For pyocyanin the $\mathrm{R}_{f}$ value 0.53 , compared with reference $\mathrm{R}_{f}$ from $P$. aeruginosa PAIO [21] and P. aeruginosa TISTR 781 [22] respectively. In solvent system, acetonitrile: methanol: water $\mathrm{R}_{f}$ value 0.88 was found for $2-4$, DAPG, which resemble to reference $\mathrm{R}_{f}$ value for $P$. fluorescens $7-14$. Similarly $\mathrm{R}_{f}=0.77$ for pyoluteorin and $\mathrm{R}_{f}=0.28$ and 0.23 for pyrrolnitrin were calculated with the reference value for $P$. cepacia strain In-b-6854 and P. cepacia strain In-b-6858 [21], respectively. PCN 
was detected on $\mathrm{R}_{f}=0.47$ with reference of $P$. chlororaphis PCL1391 [23] in the solvent system hexane: ethyl acetate. On the other hand, the strain B. cepacia UPMB3 absorbed and appeared as blue bands in solvent system acetonitrile: methanol: water for pyoluteorin, pyrrolnitrin and pyocyanin with the $\mathrm{R}_{f}$ values $0.77,0.23$ and 0.53 respectively and compared with the reference $\mathrm{R}_{f}$ value by Rosales., et al. [21] and Saosoong., et al. [22].

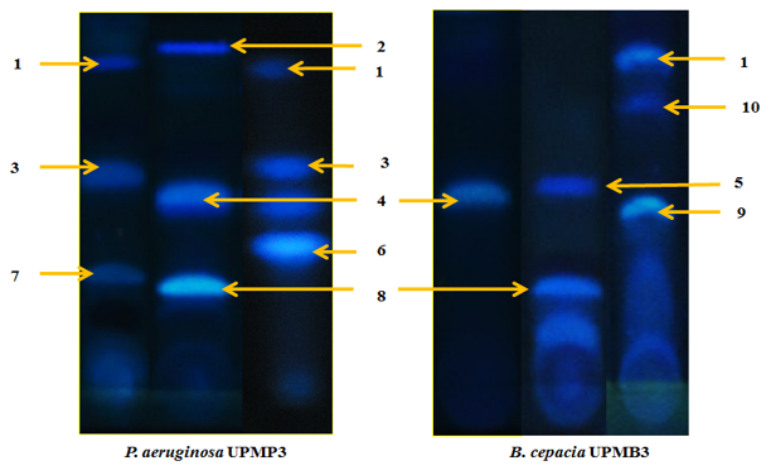

Figure 1: Detection of antibiotics produced by P. aeruginosa UPMP3 and B. cepacia UPMB3 on TLC plate. Different band indicated different antibiotics. Pyoluteorin (0.77) (1); 2,4-DAPG (0.88) (2); Phenazine (0.63) (3); Pyocyanin $(0.53)(4,5)$; PCN (0.47) (6); Pyrrolnitrin $(0.27,0.23)(7,8)$; Unidentified $(9,10)$.

In this study, the bacterial extract of $P$. aeruginosa UPMP3 produced seven bands with different $\mathrm{R}_{f}$ values out of which six bands yielded six antibiotics i.e. pyoluteorin, 2, 4-DAPG, phenazine, pyocyanin, PCN and pyrrolnitrin compared with the references. This finding collaborates that of Chang and Blackwood [24] who indicated that certain strain produced more than one antibiotic. P. aeruginosa also has been reported to produce, phenazine and phenazine derivatives such as dihydroxy - phenazine -1 - carboxylic acid, PCA, chloraphine, oxychlororaphine and aeruginosin B. The obtained results were found similar to those detected by Saosoong., et al. [22] who use TLC assessment to purify phenazine with $\mathrm{R}_{f}$ 0.70. Hassanein., et al. [25] used TLC technique for identification of phenazine produced by $P$. aeruginosa as antifungal compounds, and Saosoong., et al. [22] used TLC as primary step for isolation and analysis of antibacterial substance produced by P. aeruginosa.
A number of Pseudomonas strains have been shown to produce phloroglucinol pyrrolnitrin, a potent antifungal compound, was isolated from P. cepacia, P. chlororaphis, P. fluorescence and P. aureofaciens [26]. On the other hand, the strain B. cepacia UPMB3 produced three antibiotics compared with the references i.e. pyoluteorin, pyocyanin and pyrrolnitrin. Dikin., et al. [27] reported that antimicrobial substances produced by $B$. cepacia developed five bands on TLC sheets with different $\mathrm{R}_{f}$ values. It was also reported that B. cepacia produces several antibiotics, most of which have antifungal activity, such as pryrrolnitrin [28] and cepacidine A [29]. Thus, the result of this study revealed that at least several active antifungal compounds are produced by the oil palm rhizosphere associated bacteria P. aeruginosa UPMP3 and B. cepacia UPMB3.

\section{Detection of siderophores}

The modified universal CAS agar plates were used for the detection of siderophores produced by P. aeruginosa UPMP3 and B. $c e$ pacia UPMB3. The results of this experiment revealed that the both bacterial strains produced siderophores with colour change blue to orange. When the bacterial strains cultured on only CAS agar petriplates, a very little response was observed in respect to the rate of growth, colour change and rate of CAS reaction (Figure 2A and 2B, Table 1). On the other hand, both the bacterial strains produced siderophores when cultured on the medium NA + CAS and KB + CAS. It was observed that in NA + CAS medium both the strains produced a little area of colour change (Figure 2D and 2E, Table 1). It was found that the bacterial strains $P$. aeruginosa UPMP3 and $B$. cepacia UPMB3 produced a maximum area of colour change (blue to orange) in $\mathrm{KB}+\mathrm{CAS}$ medium after 21 days of culture respectively (Figure $2 \mathrm{G}$ and $2 \mathrm{H}$, Table 1 ). No colour change indicated no siderophores production on control plates (Figure 2C, 2F, and 2I; Table 1). It was observed that both P. aeruginosa UPMP3 and B. cepacia UPMB3 produced more siderophores when cultured on KB medium.

Rhizobacteria belonging to the genera Azospirillum, Bacillus, Pseudomonas, Xanthomonas, Burkholderia and Rhizobium have been shown to produce different types of siderophores which help in stimulating plant growth as well as control of plant pathogens. Siderophores-producing bacteria have been used as biocontrol agents to combat plant pathogens. Different rhizosphere bacteria produce different types of siderophores. Siderophores produced by Pseudomonas sp. have been employed efficiently as biocontrol 


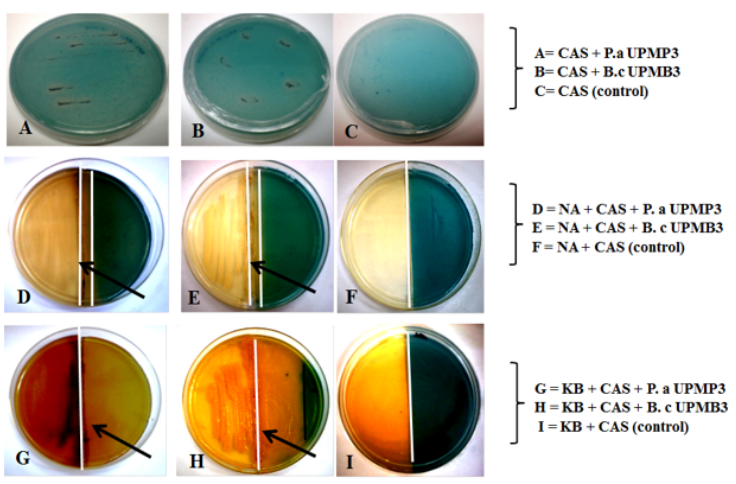

Figure 2: Siderophores production by $P$. aeruginosa UPMP3 and B. cepacia UPMB3 in different media supplemented with CAS agar at 21 days.

\begin{tabular}{|l|c|c|c|c|}
\hline \multirow{4}{*}{$\begin{array}{c}\text { Bacterial } \\
\text { strain }\end{array}$} & Media & \multicolumn{3}{|c|}{$\begin{array}{r}\text { Degree of colour change/ } \\
\text { siderophores production after } \\
\mathbf{7 , 1 4} \text { and 21 days }\end{array}$} \\
\cline { 2 - 5 } & & $\mathbf{7}$ & $\mathbf{1 4}$ & $\mathbf{2 1}$ \\
\hline \multirow{3}{*}{$\begin{array}{l}\text { P. aeruginosa } \\
\text { UPMP3 }\end{array}$} & $\mathrm{CAS}$ & - & - & + \\
\cline { 2 - 5 } & $\mathrm{NA}+\mathrm{CAS}$ & - & + & + \\
\cline { 2 - 5 } & $\mathrm{KB}+\mathrm{CAS}$ & ++ & +++ & ++++ \\
\hline \multirow{3}{*}{$\begin{array}{l}\text { B. cepacia } \\
\text { UPMB3 }\end{array}$} & $\mathrm{CAS}$ & - & - & - \\
\cline { 2 - 5 } & $\mathrm{NA}+\mathrm{CAS}$ & - & + & + \\
\cline { 2 - 5 } & $\mathrm{KB}+\mathrm{CAS}$ & + & ++ & +++ \\
\hline
\end{tabular}

Table 1: Degree of siderophores production by $P$ aeruginosa UPMP3 and B. cepacia UPMB3 after 21 days.

- = Negative, $+=$ Positive $(+=25 \%,++=50 \%,+++=75 \%$, $++++100 \%)$

agents against soil borne plant pathogen. $P$. aeruginosa produces two types of siderophores, phyochelin and pyoverdine which have been widely studied as biological mechanism in the control of phyto pathogenic fungi and bacteria [30]. It has been demonstrated that one strain can produce more than one type of siderophores, thus Burkholderia cepacia strains produce different types of siderophores, namely ornibactin and cepaciachelin, hydroxamateand catecholate-type siderophores, respectively [31]. This ability to produce siderophores has also been commonly associated with the capacity of biocontrol shown by Pseudomonas fluorescens strains. Martha., et al. [32] reported that Pseudomonas aeruginosa, P. putida biovar B, P. marginalis and Burkholderia cepacia, isolated from the rhizosphere and the phyllosphere of rose and alstroemeria plants identified by biochemical assays and cultured in King's B medium, showed antagonistic properties in vitro against the pathogen Rhizoctonia solani and Botrytis cinerea coincided with the presence of siderophores. B. subtilis QM3, a spore-forming bacterium commonly used in commercial and research bio-control products to control a variety of plant pathogens [33], was proved to be a siderophores producer using universal CAS assay and presented distinct orange halos on SD-CAS assay. This indicated an iron chelator removed iron from the blue CAS complex thus causing its color change to orange.

\section{Detection of HCN}

For the detection of HCN the both bacterial strains were grown in KB broth with glycine and incubated for varying time periods ( 0 to 10 days). HCN released into the nutrient broth was observed at intervals of 24 hours. The results of this study revealed that the strain P. aeruginosa UPMP3 was found to be strong HCN producers by changing yellow colour of the filter paper with a trend to dark brown to red colour (Figure 3A). On the other hand, B. cepacia UPMB3 did not produce HCN without any colour change of the filter paper (Figure 3C) as the control (Figure 3B).

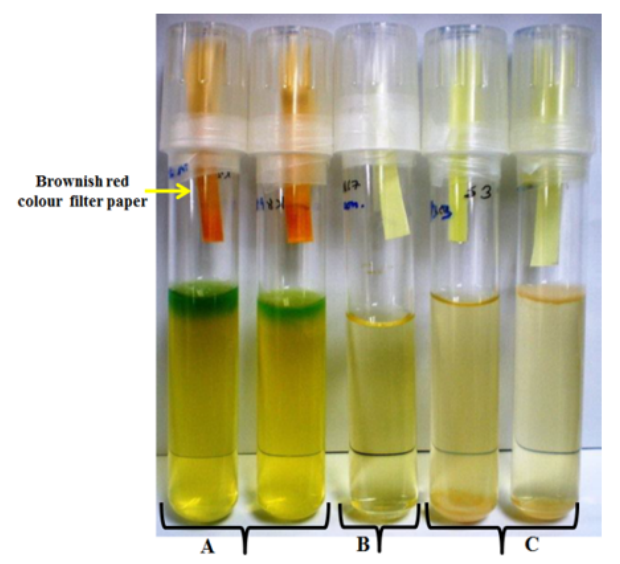

Figure 3: Detection of HCN production by $P$. aeruginosa UPMP3 and B. cepacia UPMB3 after 10 days of incubation. $\mathrm{A}=P$. aeruginosa UPMP3, B = Control (un-inoculated $\mathrm{KB}$ broth), $\mathrm{C}=B$. cepacia UPMB3. 
Hydrogen cyanide (HCN) is an example of a metabolite that can differentially affect plant growth depending on the producer stains in rhizosphere. $\mathrm{HCN}$ production by antagonistic rhizobacteria plays an important role in the biological control of pathogens as well as enhance plant establishment. In Pseudomonad species, HCN is released by the decarboxylation of glycine and formed a brownish red compound with sodium picrate and the intensity of the colour increases with the amount of HCN. It has been reported that glycine is a carbon precursor for HCN in P. aeruginosa HCN effectively blocks the cytochrome oxidase pathway and is highly toxic to all aerobic microorganisms at picomolar concentrations. However, producer microbes, mainly Pseudomonads, are reported to be resistant [34]. The production of HCN by Pseudomonas cf. monteilii 9 which turned yellow colour filter strip to reddish colour that played a contributory role in inhibition of pathogens, S. rolfsii [35]. The HCN production is found to be a common trait of Pseudomonas (88.89\%) and Bacillus (50\%) in the rhizospheric soil and plant root nodules [12] and is a potential and environmentally compatible mechanism for biological control of weeds.

\section{Effect of bacterial antibiotics against $G$. boninense}

In bioassay test both bacterial strains and Ganoderma were cultured in King's B medium. From the result it was observed that the Ganoderma mycelial growth was normal in control plate after seven days of incubation similar to that in MEA medium. In this experiment, both bacterial strains $P$. aeruginosa UPMP3 and B. cepacia UPMB3 were found to produce diffusible antibiotics which showed a significant effect to control the Ganoderma mycelial growth in vitro after 2, 4 and 7 days of inoculation (Figure 4). The results revealed that the strain $P$. aeruginosa UPMP3 showed the highest (94.21\%) inhibitory effect on the mycelial growth of $G$. boninense after 7 days of incubation (Figure 6). On the other hand, B. cepacia UPMB3 showed maximum (21.27\%) inhibition compared to control treatment (Figure 6). From the result it was observed that the average growth of $G$. boninense was higher in control treatment 8.6 $\mathrm{cm}$ after 7 days. Whereas no mycelial growth was observed when $G$. boninense cultured with $P$. aeruginosa UPMP3 in compared to culture with B. cepacia UPMB3 (Figure 5). It was found that P. aeruginosa UPMP3 was more effective than B. cepacia UPMB3 to control G. boninense in vitro.

Bergsma-Vlami., et al. [36] demonstrated in vitro and in vivo production of antibiotics by numerous antifungal bacterial strains. Several authors have been reported involvement of antibiosis in

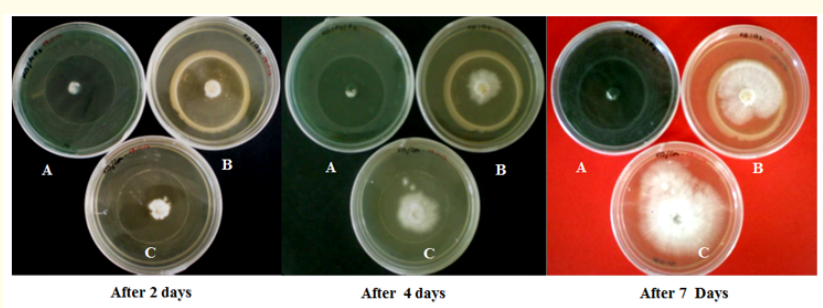

Figure 4: Bio assay test for the production of antibiotic substances by $P$. aeruginosa UPMP3 and B. cepacia UPMB3 against $G$. boninense on $\mathrm{KB}$ medium after 7 days of incubation. $\mathrm{A}=P$. aeruginosa $\mathrm{UPMP} 3+G$. boninense, $\mathrm{B}=$ B. cepacia $\mathrm{UPMB} 3+G$. boninense, $\mathrm{C}=$ control.

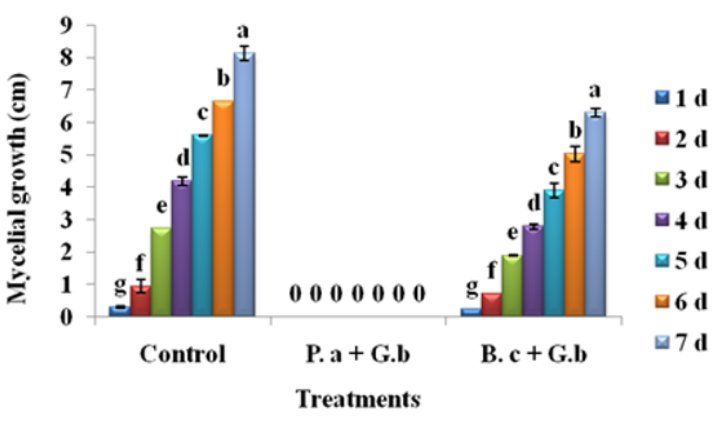

Figure 5: Effect of antibiotic substances on mycelial growth of Ganoderma in different treatments after 1-7 days of incubation. P.a = Pseudomonas aeruginosa UPMP3, B.c = Burkholderia cepacia UPMB3, G.b = Ganoderma boninense. Values followed by the same letter are not significantly different according to LSD test at $\mathrm{P}<0.05$ level. Each value is the mean of 3 replications.

Vertical bars represent standard error.

biocontrol of plant pathogens. Mechanism of anti-fungal activities by the biocontrol agents Streptomyces violaceusniger strain G10 on Fusarium oxysporum f. sp. cubense race and Pantoea agglomerans strain Eh252 on Erwinia amylovora [37] and the biocontrol of Pythium damping off of Pea by Burkholderia cepacia [38] was attributed to antibiosis. Rakh., et al. [35] reported that the Pseudomonas 


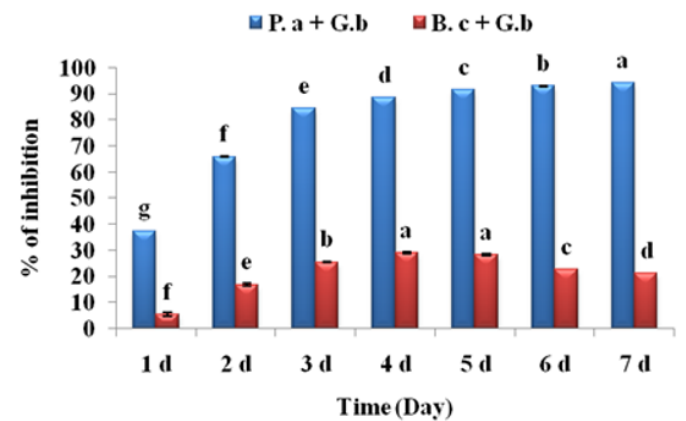

Figure 6: Inhibitory effects of antibiotics produced by P. aeruginosa UPMP3 and B. cepacia UPMB3 on mycelial growth of $G$. boninense after 1-7 days. P.a = Pseudomonas aeruginosa UPMP3, B.c = Burkholderia cepacia UPMB3, G.b = Ganoderma boninense. Values followed by the same letter are not significantly different according to LSD test at $\mathrm{P}<0.05$ level. Each value is the mean of

3 replications. Vertical bars represent standard error.

cf. monteilii 9 strain was able to produce non-volatile diffusible metabolites and inhibits the mycelial growth of Sclerotium rolfsii causing stem rot of groundnut in King's B medium. Fluorescent pseudomonads have been reported as promising biological control agents against $S$. rolfsii in betelvine [39] and bean [40] among others. Manwar., et al. [41] reported that the antibiotic pyocyanin and pyoverdine produced by Pseudomonas inhibited the growth of Aspergillus niger under in vitro conditions. This showed the importance of antibiotics production in the control of deleterious soilborne pathogens by PGPR strains. These results are in accordance with that $P$. aeruginosa UPMP3 and B. cepacia UPMB3 produced non-volatile diffusible antibiotics which inhibited $G$. boninense. 2-4-Diacetylphloroglucinol, the best known Phl compound, causes membrane damage to Pythium spp. and is particularly inhibitory to zoospores of this oomycete [1]. Dikin., et al. [42] reported that pyrrolnitrin causes the loss of mitochondrial activity in the fungal cytoplasm, inhibiting succinate oxidase and NADH-cytochrome reductase. Pyrrolnitrin also interferes with cellular processes such as oxidative stress, blockage of electron transport as well as inhibition of DNA and RNA synthesis.

Effect of volatile and non-volatile metabolites against $G$. boninense in vitro

Both the bacterial strains $P$. aeruginosa UPMP3 and B. cepacia UPMB3 were found to produce volatile and non-volatile substances which suppressed Ganoderma mycelial growth in vitro after different days of inoculation (Figure 7). From the result it was observed that the strain P. aeruginosa UPMP3 showed the highest inhibitory effect $51.16 \%$ of $G$. boninense mycelial growth and $B$. cepacia UPMB3 showed maximum $8.89 \%$ inhibition compared to control treatment after 7 days of incubation respectively (Figure 9). The result revealed that the average growth of $G$. boninense was higher in control treatment i.e. $6.86 \mathrm{~cm}$ after 7 days which was near about the average growth of $G$. boninense with B. cepacia UPMB3 treatment $(6.25 \mathrm{~cm})$ (Figure 8).

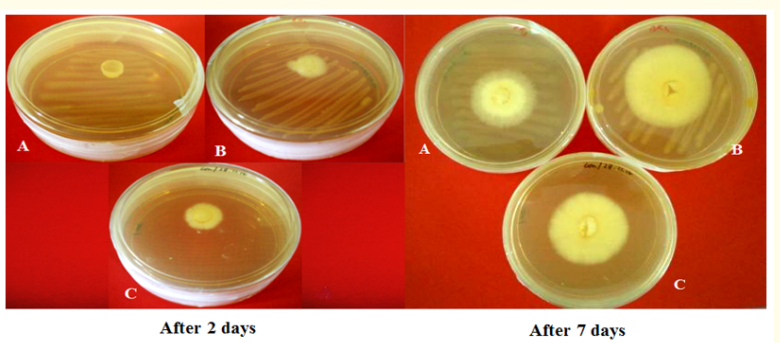

Figure 7: Bio assay test for the production of volatile and non-volatile substances by $P$. aeruginosa UPMP3 and B. cepacia UPMB3 against $G$. boninense after 7 days of incubation. $A=P$. aeruginosa $\mathrm{UPMP} 3+G$. boninense, $\mathrm{B}=$ B. cepacia $\mathrm{UPMB} 3+G$. boninense, $\mathrm{C}=$ control.

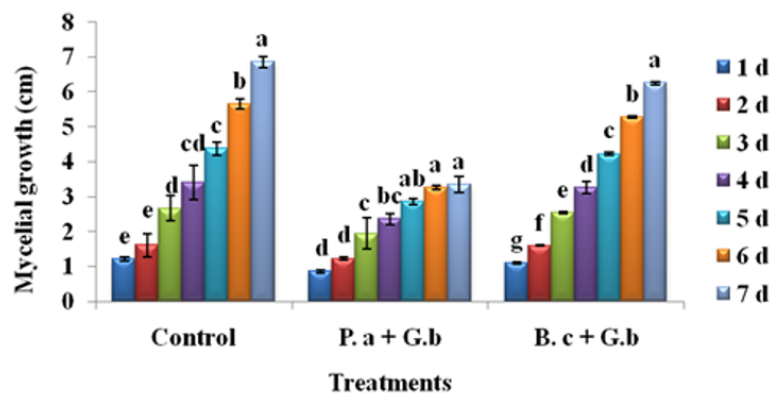

Figure 8: Effect of volatile and non-volatile substances on mycelial growth of Ganoderma in different treatments after 7 days of incubation. P.a = Pseudomonas aeruginosa UPMP3, B.c = Burkholderia cepacia UPMB3, G.b = Ganoderma boninense. Values followed by the same letter are not significantly different according to LSD test at $\mathrm{P}<0.05$ level. Each value is the mean of 3 replications. Vertical bars represent standard error. 


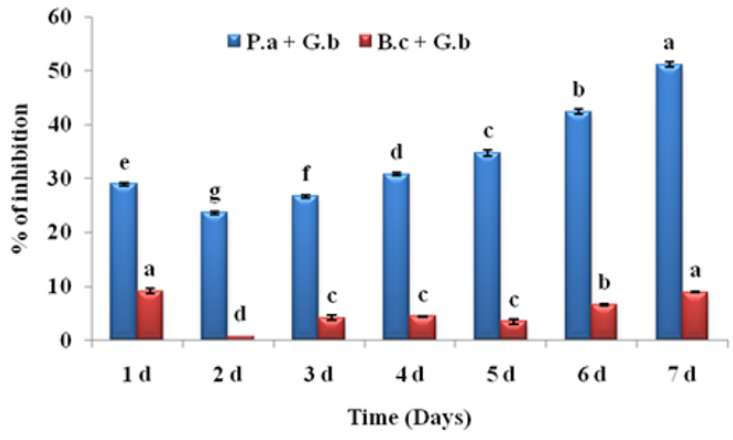

Figure 9: Inhibitory effects of volatile and non-volatile substances produced by P. aeruginosa UPMP3 and B. cepacia UPMB3 on mycelial growth of $G$. boninense after 7 days of incubation. P.a = Pseudomonas aeruginosa UPMP3, B.c = Burkholderia cepacia UPMB3, G.b = Ganoderma boninense. Values followed by the same letter are not significantly different according to LSD test at $\mathrm{P}<0.05$ level. Each value is the mean of

3 replications. Vertical bars represent standard error.

On the other hand, the average growth for the treatment $G$. boninense with $P$. aeruginosa UPMP3 was $3.35 \mathrm{~cm}$ (Figure 8 ). From the above result it was reported that $P$. aeruginosa UPMP3 was more capable to produced volatiles and non-volatile substances than B. cepacia UPMB3.

The mechanism of antibiosis by the antagonistic rhizobacteria and inhibition of pathogens was more pronounced through volatile and non-volatile metabolites including aldehydes, alcohols, ketones and sulphides. Volatile toxic substances produced by antagonists could diffuse easily and inhibited the growth of the pathogen in vitro and even in soil they easily diffuse through the air filled pores of the soil and inhibited the soil-borne pathogens. The production of volatile metabolites, components and inhibition of the test pathogen by volatile metabolites varied among different PGPR. Ramettee., et al. [43] reported that hydrogen cyanide is a broad spectrum antimicrobial compound involved in biological control of root disease by many plant associated fluorescent pseudomonads. Production of HCN by certain strains of fluorescent pseudomonads has been involved in the suppression of soil borne pathogens.

The antifungal nature of the organic volatiles has been demonstrated in several pathogen systems such as inhibition of hyphal ex- tension, inhibition of carpogenic germination of sclerotia of $S$. sclerotiorum in bean by allyl alcohol and control of root rot of tobacco by hydrogen cyanide produced by pseudomonads [44]. Besides, aliphatic aldehydes and ketones were more effective than alcohols in the post-harvest control of grey mould caused by Botrytis cinerea [45] and in the inhibition of germ tube formation of Alternaria alternata [46].

Moreover, Pseudomonas sp. are capable of producing organic volatiles whose in vitro antifungal nature has been demonstrated against Phytophthora vignae in cowpea [47]. It was also reported that Burkholderia cepacia B23 producing volatile metabolites was effective to control the post-harvest anthracnose disease of papaya caused by Colletotrichum gloeosporioides whose inhibition percentage was 26.61 [48]. Diby., et al. [49] reported that Pseudomonas $c f$. monteilii 9 produced volatile metabolites which inhibited $S$. rolfsii $(100 \%)$ as well as it controlled Pythium ultimum and Phytophthora capsici. In accordance with the result of the present experiments, the bacterial strains $P$. aeruginosa UPMP3 is more effective to produce volatile and non-volatile substances and suppress the growth of $G$. boninense than B. cepacia UPMB3 in vitro. Watanabe., et al. [50] reported that Burkholderia sp. isolate 87-11 obtained from basidiospore of Lentinus lepideus, showed antagonistic activity against Pythium aphanidermatum and Rhizoctonia solani. Production of pyrrolnitrin pyoluteolin, cepabactin, volatile ammonia and siderophore are the major mechanisms proposed for the disease suppressive effects of $B$. cepacia.

\section{Conclusion}

The present study strongly support that the antimicrobial metabolites may be used in the management of microbial/fungal infection and the finding highlights the importance for further investigation towards the goal of obtaining novel antimicrobial agent. Although the bacterial strains obtained in this study cannot be declared as new antibiotics, there is the probability of finding new antibiotics in oil palm rhizosphere because of its wide biodiversity. Further analysis of compounds by spectrometric, chromatographic techniques and strain improvement studies provides detailed information about the compounds produced by the bacterial strains and the ability of the strains in producing newer compounds. Besides, exploitation of PGPR-plant interactions can result in the promotion of plant health and can play a significant role in low-input sustainable agriculture applications for commercial crops. 


\section{Acknowledgements}

The authors would like to thank Ministry of Higher Education (MOHE) Malaysia for providing the Fundamental Research Grant Scheme (FRGS) to carry out this study.

\section{Conflict of Interest}

All authors declare no conflict of interest.

\section{Bibliography}

1. De Souza JT., et al. "Frequency, diversity, and activity of 2,4-diacetylphloroglucinol-producing fluorescent Pseudomonas spp. in Dutch take all decline soils". Phytopathology 93.1 (2003): 54-63.

2. Raaijmakers JM., et al. "Antibiotic production by bacterial bio control agents". Antonie Van Leeuwenhoek International Journal 81.1-4 (2002): 537- 547.

3. Weller DM and Cook RJ. "Suppression of take-all of wheat by seed treatment with fluorescent pseudomonads". Phytopathology 73.3 (1983): 463-469.

4. Parvin W., et al. "Detection of phenazines from UPMP3 strain of Pseudomonas aeruginosa and its antagonistic effects against Ganoderma boninense". International Journal of Agriculture and Biology 18.3 (2016): 483-488.

5. Ownley BH., et al. "Identification and manipulation of soil properties to improve the biological control performance of phenazine-producing Pseudomonas fluorescens". Applied and Environmental Microbiology 69.6 (2003): 3333-3343.

6. Petterson M and Baath E. "Effects of the properties of the bacterial community on $\mathrm{pH}$ adaptation during recolonization of a humous soil". Soil Biology and Biochemistry 36.9 (2004): 13831388.

7. Picard C., et al. "Frequency and biodiversity of 2, 4- diacetylphloroglucinol -producing bacteria isolated from the maize rhizosphere at different stages of plant growth". Applied and Environmental Microbiology 66.3 (2000): 948-955.

8. Lodewyckx CJ., et al. "Endophytic bacteria and their potential applications". Critical Reviews in Plant Sciences 21.6 (2002): 583-606
9. Hernandez ME., et al. "Phenazines and other redox-active antibiotics promote microbial mineral reduction". Applied and Environmental Microbiology 70.2 (2004): 921-928.

10. Duijff BJ., et al. "Siderophore mediated competition for iron and induced resistance in the suppression of Fusarium wilt of carnation by Fluorescent Pseudomonas spp". Netherlands Journal of Plant Pathology 99.9 (1993): 277-289.

11. Siddiqui IA., et al. "Role of cyanide production by Pseudomonas fluorescens $\mathrm{CHAO}$ in the suppression of root-knot nematode, Meloidogyne javanica in tomato". World Journal of Microbiology and Biotechnology 22.3 (2006): 641-650.

12. Ahmad F., et al. "Screening of free-living rhizospheric bacteria for their multiple plant growth promoting activities". Microbiological Research 163.2 (2008): 173-181.

13. Dowling DN and O'gara F. "Metabolites of Pseudomonas involved in the bio control of plant disease". Trends in Biotechnology 12.4 (1994): 133-141.

14. Zaiton S., et al. "Isolation and Characterization of Microbial Endophytes from Oil Palm Roots: Implication as Biocontrol Agents against Ganoderma". The Planter 82.966 (2006): 587597.

15. Azadeh BF. and Sariah M. "Molecular characterization of Pseudomonas aeruginosa UPMP3 from oil palm rhizosphere". American Journal of Applied Sciences 6.11 (2009): 1915 -1919.

16. Bernal G., et al. "Isolation and partial purification of a metabolite from a mutant strain of Bacillus sp. with antibiotic activity against plant pathogenic agents". Electronic Journal of Biotechnology 5.1 (2002): 01-09.

17. Schwyn B and Neilands JB. "Universal Chemical Assay for the Detection and Determination of Siderophores". Analytical Biochemistry 160.1 (1987): 47-56.

18. Reddy BP., et al. "Efficacy of antimicrobial metabolites of Pseudomonas fluorescens against rice fungal pathogens". Current Trends in Biotechnology and Pharmacy 2.1 (2008): 178-182.

19. Montealegro JR., et al. "Selection of bio antagonistic bacteria to be used in biological control of Rhizoctonia solani in tomato". Electronic Journal of Biotechnology 6.2 (2003): 115-127. 
20. El-Sayed W., et al. "Isolation and Identification of Phenazine1-Carboxylic acid from different Pesudomonas isolates and its Biological activity against Alternaria solani”. Research Journal of Agriculture and Biological Sciences 4.6 (2008): 892-901.

21. Rosales AM., et al. "Isolation and identification of antifungal metabolites produced by rice associated antagonistic Pseudomonas spp". Phytopathology 85.9 (1995): 1028-1032.

22. Saosoong K., et al. "Isolation and Analysis of Antibacterial Substance Produced from P. aeruginosa TISTR 781". Khon Kaen University Science Journal 37.2 (2009): 163-172.

23. Thomas FC., et al. "Introduction of the phzH Gene of Pseudomonas chlororaphis PCL1391 Extends the Range of Biocontrol Ability of Phenazine-1-Carboxylic Acid-Producing Pseudomonas spp. Strains". Molecular Plant-Microbe Interactions 14.8 (2001): 1006-1015.

24. Chang PC and Blackwood AC. "Simultaneous production of three phenazine pigments by Pseudomonas aeruginosa Mac 436". Canadian Journal of Microbiology 15.5 (1969): 439-444.

25. Hassanein WA., et al. "Characterization and antagonistic activates of Metabolite produced by P. aeruginosa Sha8". Journal of Applied Sciences Research 5.4 (2009): 392-403.

26. Keel C., et al. "Suppression of Root Diseases by Pseudomonas fluorescens CHA0: Importance of the Bacterial Secondary Metabolite 2,4-Diacetylphloroglucinol". Molecular Plant-Microbe Interactions 5.1 (1992): 4-13.

27. Dikin A., et al. "Extraction of antimicrobial substances from antagonistic bacteria against Schizophyllum commune" Fr. In: Proceedings, 27th Symposium of the Malaysian Society for Microbiology, 24-27 November 2005. Grand Plaza Parkroyal, Penang, Malaysia. (2005).

28. Hwang J., et al. "Pyrrolnitrin production by Burkholderia cepacia and biocontrol of rhizoctonia stem rot of poinsettia". Biological Control 25.1 (2002): 56-63.

29. Lee $\mathrm{CH}$., et al. "Cepacidine A, a novel antifungal antibiotic produced by Pseudomonas cepacia. I. Taxonomy, production, isolation and biological activity". The Journal of Antibiotics 47.12 (1994): 1402-1405.
30. Sajeed SA and Vidhale NN. "Antagonistic Activity of Siderophore Producing Pseudomonas aeruginosa against Aspergillus Spp. and Candida albicans". Research Journal of Pharmaceutical, Biological and Chemical Sciences 3.4 (2012): 719-726.

31. Katy Di'az P., et al. "Production of phytohormones, siderophores and population fluctuation of two root-promoting rhizobacteria in Eucalyptus globulus cuttings". World Journal of Microbiology and Biotechnology 28.5 (2012): 2003-2014.

32. Martha P., et al. "Siderophore Producing Pseudomonas as Pathogenic Rhizoctonia solani and Botrytis cinerea Antagonists". Universitas Scientiarum 10.1 (2005): 65-74.

33. Hu Q-P., et al. "Isolation and identification of a potential bio control agent Bacillus subtilis QM3 from Qinghai yak dung in China". World Journal of Microbiology and Biotechnology 24.11 (2008): 2451-2458.

34. Castric PA. "Glycine Metabolism by Pseudomonas aeruginosa: Hydrogen Cyanide Biosynthesis". The Journal of Bacteriology 130.2 (1977): 826-831.

35. Rakh RR., et al. "Biological Control of Sclerotium rolfsii, Causing Stem Rot of Groundnut by Pseudomonas cf. monteilii 9". Recent Research in Science and Technology 3.3 (2011): 26-34.

36. Bergsma-Vlami M., et al. "Assessment of Genotypic Diversity of Antibiotic-Producing Pseudomonas Species in the Rhizosphere by Denaturing Gradient Gel Electrophoresis". Applied and Environmental Microbiology 71.2 (2005): 993-1003.

37. Stockwell VO., et al. "Antibiosis contributes to biological control of fire blight by Pantoea agglomerans strain Eh252 in orchards". Phytopathology 92.11 (2002): 1202-1209.

38. Heungens $\mathrm{K}$ and Parke JL. "Post infection biological control of oomycete pathogens of pea by Burkholderia cepacia AMMDR1". Phytopathology 91.4 (2001): 383-391.

39. Singh A., et al. "Biocontrol of collar rot disease of betelvine (Piper betle L.) caused by Sclerotium rolfsii by using rhizosphere-competent Pseudomonas fluorescens NBRI-N6 and $P$. fluorescens NBRI-N". Current Microbiology 47.2 (2003): 153158. 
40. De La Fuente L., et al. "Pseudomonas fluorescens UP61 isolated from birdsfoot trefoil rhizosphere produces multiple antibiotics and exerts a broad spectrum of biocontrol activity". European Journal of Plant Pathology 110.7 (2004): 671-681.

41. Manwar AV., et al. "Siderophore production by a marine Pseudomonas aeruginosa and its antagonistic action against phytopathogenic fungi". Applied Biochemistry and Biotechnology 118.1-3 (2004): 243-252.

42. Dikin A., et al. "Mode of action of antimicrobial substances from Burkholderia multivorans and Microbacterium testaceum against Schizophyllum commune Fr". International Journal of Agriculture and Biology 9.2 (2007): 311-314.

43. Ramette A., et al. "Prevalence of fluorescent pseudomonads producing antifungal phloroglucinols and/or hydrogen cyanide in soils naturally suppressive or conducive to tobacco root rot". FEMS Microbiology Ecology 44.1 (2003): 35-43.

44. Voisard C., et al. "Cyanide production by Pseudomonas fluorescens helps suppress black root rot of tobacco under gnotobiotic conditions". The EMBO Journal 8.2 (1989): 351-358.

45. Archibold DD., et al. "Identifying natural volatile compounds that control gray mold (Botrytis cinerea) during postharvest storage of strawberry, blackberry and grape". Journal of Agricultural and Food Chemistry 45.10 (1997): 4032-4037.

46. Andersen RA., et al. "Structure-antifungal activity relationships among C6 and C9 aliphatic aldehydes, ketones and alcohols". Journal of Agricultural and Food Chemistry 42.7 (1994): 1563-1568.

47. Fernando WGD and Linderman RG. "Inhibition of Phytophthora vignae and root rot of cowpea by soil bacteria”. Biological Agriculture and Horticulture 12.1 (1994): 1-14.

48. Rahman MA., et al. "Screening of antagonistic bacteria for biocontrol activities on Colletotrichum gloeosporioides in papaya". Asian Journal of Plant Sciences 6.1 (2007): 12-20.

49. Diby P., et al. "Antagonistic mechanisms of fluorescent Pseudomonas against Phytophthora capsici Leonian in black pepper (Piper nigrum Linn.)". Journal of Spices and Aromatic Crops 14.2 (2005): 94-101.
50. Watanabe T., et al. "Potential of antago-nistic isolate obtained from Lentinus lepideus basidiospores as a biocontrol agent". Mycoscience 41.1 (2000): 79-82.

\section{Volume 5 Issue 9 September 2021 \\ (C) All rights are reserved by Waheeda Parvin., et al.}

